\title{
LA CREACIÓN LÉXICA EN UNA LENGUA ARTIFICIAL: EL DOTHRAKI, DE JUEGO DE TRONOS
}

\author{
THE LEXICAL CREATION IN AN ARTIFICIAL LANGUAGE: \\ THE DOTHRAKI, OF GAME OF THRONES
}

\author{
Leticia GÁndara Fernández \\ Universidad de Extremadura \\ leticiagf@unex.es \\ https://orcid.org/0000-0001-6360-0722
}

Recibido: $17 / 10 / 2019$

Aceptado: 28/04/2020

\begin{abstract}
Resumen
Este trabajo presenta un análisis del léxico del dothraki, lengua inventada para la popular adaptación televisiva Juego de tronos, basada en la saga Canción de hielo y fuego de George R. R. Martin. Primeramente, el estudio se ha centrado en los términos inventados por Martin con el fin de observar cómo estos influyen decisivamente en la construcción del vocabulario del dothraki. En segundo lugar, se incluye un corpus de palabras pertenecientes a los campos léxicos y semánticos más importantes de esta lengua. Estos permiten trazar, en último lugar, un análisis de los procedimientos de formación léxica empleados por David J. Peterson en el diseño de este sistema lingüístico artificial.

PALABRAS CLAVE: léxico; lengua artificial; dothraki; Canción de hielo y fuego; Juego de tronos.
\end{abstract}

\begin{abstract}
This paper presents an analysis of the lexicon of Dothraki, a language invented for the popular television adaptation Game of Thrones, based on the saga Song of Ice and Fire by George R. R. Martin. First, the study has focused on the terms invented by Martin in order to observe how they influence decisively the construction of Dothraki vocabulary. Secondly, a corpus of words belonging to the most important lexical and semantic fields of this language is included. These allow, finally, to draw an analysis of the lexical training procedures employed by David J. Peterson in the design of this artificial linguistic system.

KEYWORDS: lexicon; artificial language; dothraki; Song of Ice and Fire; Game of Thrones.
\end{abstract}

Para citar este artículo / To cite this article: Gándara Fernández, Leticia (2020). La creación léxica en una lengua artificial: el dothraki, de Juego de Tronos. ELUA, 34: 95-116. https://doi.org/10.14198/ ELUA2020.34.4

Enlace / Link: https://doi.org/10.14198/ELUA2020.34.4 


\section{INTRODUCCIÓN}

Desde sus inicios, el movimiento de creación de sistemas lingüísticos artificiales ha atravesado diferentes etapas, acompañado inevitablemente de una evolución interna en sus motivaciones y en sus resultados, consecuencia del marco socio-cultural de cada periodo histórico (Martínez 2016: 78). Los motivos que propiciaron la elaboración de los primeros diseños artificiales poco tienen que ver con los intereses por los que actualmente se siguen diseñando lenguas ${ }^{1}$. Los proyectos lingüísticos relevantes del XVII obedecían a "motivos científicos o filosóficos, tales como el deseo de garantizar la transparencia, racionalidad y univocidad de la comunicación científica mediante nuevos sistemas con proyección universal" (Galán 2018: 75). Estos se construyeron sin tener en cuenta las lenguas naturales; de ahí que Couturat y Leau las denominen lenguas a priori (1903: xxxvii). Dependiendo de su diseño y de sus pretensiones filosóficas, se dividen en pasigrafías (códigos universales escritos) y pasifrasías o lenguas a priori propiamente dichas (sistemas lingüísticos pensados para ser utilizados tanto en la oralidad como en la escritura, aunque continúan dando prioridad a esta última) ${ }^{2}$. Sus creadores, caracterizados por Yaguello (1984) como lunáticos del lenguaje, pretendían diseñar lenguas lógicas, perfectas y regulares, de uso restringido para hombres de ciencia (Calero 2010: 18). La finalidad científica que domina en este tipo de proyectos dio paso en el siglo XIX a una motivación pragmática. Se produjo así un cambio en el paradigma de la creación de lenguas que propició la aparición de otro tipo de sistemas, denominados por Couturat y Leau (1903) lenguas a posteriori. A diferencia de las anteriores, que también tienen cabida en pleno siglo XIX, estas se plantean como auténticos códigos de comunicación universal. Con el fin de conseguir su fácil y rápido aprendizaje, los autores se basan en los elementos más comunes de los idiomas europeos más extendidos para construir sus lenguas. En este momento, no se considera prioritario disponer de un instrumento de la razón capaz de reflejar con exactitud y fiabilidad el pensamiento humano, sino una verdadera herramienta de comunicación universal -sencilla, neutra y racional- que facilite el contacto entre personas de lugares distintos (Grande 2008: 111).

La intensa labor lingüística llevada a cabo por los inventores de lenguas de siglos precedentes tiene como resultado una lista interminable de proyectos diferentes pero con carac-

1 Para una aproximación a la historia de las lenguas artificiales, véase Couturat y Leau (1903), Eco (1994) y Calero (1999).

2 Según Couturat y Leau (1903: xxviii), podemos diferenciar dos subgrupos dentro de las lenguas a priori atendiendo a sus pretensiones y al método utilizado para su elaboración: lenguas filosóficas y no filosóficas. Las primeras se caracterizan por su afán de establecer una clasificación lógica de los elementos de la naturaleza. Estas gozaron de un mayor éxito entre los hombre de ciencia de la época. Como ejemplos, citamos los proyectos ideados por George Dalgarno (Ars signorum, vulgo character universalis et lingua philosophica, 1661) y John Wilkins (An essay toward a real character and a philosophical language, 1668). Las segundas, por su parte, pretenden imponer una ordenación conceptual del universo. De estas, el ejemplo más significativo es el Solresol (1866) de Jean François Sudre.

3 La lengua a posteriori más conocida es el esperanto, diseñado por Luidwik Lejzer Zamenhof (1859) en 1887. Bajo el seudónimo Doctor Esperanzado o Doktoro Esperanto (de ahí el nombre de la lengua), este médico oftalmólogo demostró poseer un gran talento para los idiomas, que le permitió desarrollar un sistema lingüístico con el que sembrar la paz y la unidad entre los pueblos. Este se caracteriza por poseer una gramática simple, de tipo indoeuropeo, en la que apenas se registran excepciones. De tipo aglutinante, la formación de palabras se realiza mediante la adición de afijos. Su vocabulario es principalmente latino (Jordan 1997: 41); aproximadamente, un $75 \%$ del léxico proviene de lenguas romances, un $20 \%$ de las lenguas anglogermánicas, y el resto comprende préstamos del griego, de las lenguas eslavas, del hebreo, del árabe y del japonés, entre otras (Galán 2012: 431). 
terísticas comunes ${ }^{4}$. Estos diseños artificiales se han visto condicionados por las creencias filosóficas, culturales, científicas e incluso políticas del momento histórico en el que han sido elaborados. De ahí que Galán defina el concepto de "lengua artificial" como una "construcción semiótica diseñada artificialmente para satisfacer determinados objetivos que las lenguas naturales, constreñidas histórica y culturalmente, no pueden alcanzar" (Galán 2012: 147). En la actualidad, el interés por la invención de lenguas artificiales se ha reavivado gracias a las numerosas propuestas que han surgido en el ámbito de la ficción literaria y cinematográfica. Estas se conocen como lenguas artísticas, lenguas construidas, conlangs (constructed languages) o lenguas ficticias. Este último grupo engloba proyectos como el klingon (elaborado por Okrand en 1979 para la saga Star Trek) (véase Gándara 2019), el na 'vi (construido por Frommer en 2005 para la película Avatar) o el dothraki, objeto de estudio en este trabajo.

El dothraki es una lengua artificial diseñada para la popular serie de televisión Juego de tronos (Game of thrones), basada en la saga literaria Canción de hielo y fuego (Song of Ice and Fire) (1996-2011) de George R. R. Martin (Bayonne, Nueva Jersey, 1948). Esta última se incluye en el subgénero de la literatura fantástica, conocido como fantasía épica (Balbuena 2016), y en ella se relatan las luchas de poder de los Siete Reinos de Poniente. Martin comparte con autores de este género su vocación por construir universos imaginarios en los que recrear culturas tan atractivas y exóticas como reales para sus numerosos lectores. Generalmente, los habitantes de estos mundos (im)posibles poseen diferentes lenguas que permiten la construcción de entidades individuales o grupales distintas en estos escenarios ficticios (Barnes y Heerden 2006: 115). En Canción de hielo y fuego, Martin simula una extraordinaria diversidad lingüística al incluir múltiples referencias a supuestas lenguas ficticias, tales como el dothraki o las lenguas valyrias, que, sin embargo, no elabora. Aunque estas desempeñan importantes funciones en la trama argumental ${ }^{5}$, no es posible encontrar fragmentos de estos idiomas en la saga literaria, pues Martin no desarrolla su gramática; algo que no sucede en la adaptación televisiva, en la que se recrean diálogos completos en ellas y en las que también tienen un papel fundamental (Gándara 2018: 311). El encargado de construir la gramática y el léxico de estas lenguas inventadas fue el lingüista David J. Peterson (Long Beach, California, 1981). No obstante, Martin construyó una serie de términos que, como veremos más adelante, constituyen las bases de estos diseños lingüísticos artificiales.

4 La ingente cantidad de sistemas construidos se evidencia en obras como Histoire de la langue universelle (1903), en la que sus autores, los franceses Louis Couturat y Léopold Leau, además de establecer una clasificación en lenguas a priori, 'sistemas mixtos' y lenguas a posteriori, mencionan 19 modelos de lenguas a priori y 50 proyectos entre sistemas "mixtos" y a posteriori. Esta cifra se incrementa si tenemos en cuenta las aportaciones de algunos otros eruditos dedicados también al estudio de la invención lingüística. Monnerot-Dumaine (1960), por ejemplo, registra en su obra un total de 360 proyectos de lenguas internacionales, mientras que Knowlson (1975) cataloga más de 80 obras referidas exclusivamente a modelos de lenguas universales surgidas entre los siglos XVII y XVIII. Porset (1979), por último, hace referencia a unos 173 proyectos creados en el siglo XIX. Una obra crítica más actual y ambiciosa es el Dictionnaire des langues imaginaires de Albani/Buonarroti (2010), donde se registra un exhaustivo y variado corpus de 1100 lenguas artificiales de diversa índole: lenguas fantásticas, sagradas, experimentales, etc.

5 El tema lingüístico resulta de especial interés en el argumento de la saga, pues, por ejemplo, el aislamiento que sufre Daenerys Targaryen cuando se une a los dothraki es consecuencia directa de la barrera lingüística que se establece entre ambos. En un principio, Daenerys está siempre acompañada del caballero Ser Jorah Mormont, con quien habla la lengua común. A medida que avanza la serie, la protagonista muestra una gran facilidad para el aprendizaje de lenguas, por lo que no tardará en dominar la lengua dothraki, ganándose así el respeto y la obediencia de este pueblo. 
En el verano de 2009, los productores de la serie, Dan Weiss y David Benioff, solicitaron a la Language Creation Society un proyecto piloto para la lengua dothraki. Entre las muchas propuestas recibidas, se encontraba la de Peterson, que contaba inicialmente con trescientas páginas y varios diálogos traducidos al dothraki. Cuando Peterson recibió el encargo de construir dicha lengua, los productores tan solo le pidieron que respetara dos principios: 1) debía incorporar a su creación lingüística los términos creados por George R. R. Martin en las novelas; 2) la lengua debería provocar extrañeza al ser escuchada y, ante todo, "it to sound harsh" (Peterson 2015: 25). Conviene tener en cuenta que Peterson construyó esta lengua para una cultura descrita minuciosamente en las novelas, por lo que esta determinó el proceso de invención de la lengua.

El nombre de este idioma artificial coincide con el del pueblo dothraki, que significa literalmente men who ride, the Dothraki people (Littauer 2016: 23). Estos aparecen caracterizados como un pueblo nómada, que idolatra a los caballos como animales sagrados y a los elementos de la naturaleza. Su cultura gira en torno a los equinos, de los que obtienen todo lo necesario para su supervivencia. Se organizan jerárquicamente en hordas, conocidas como khalasares, lideradas por un khal. Su vínculo con el mundo de los caballos, el saqueo y la violencia implica que su lengua albergue un gran número de términos para referirse a estos animales, a las armas y a la guerra, como mostramos en las siguientes páginas de este estudio.

El dothraki presenta un total de 27 fonemas consonánticos, entre los que no se incluyen bilabiales oclusivas ni sordas ni sonoras. Su inventario es el siguiente:

\begin{tabular}{|c|c|c|c|c|c|c|c|}
\hline & Labial & Dental & Alveolar & Palatal & Velar & Uvular & Glottal \\
\hline Oclusiva & & $\mathrm{t}, \mathrm{d}$ & & & $\mathrm{k}, \mathrm{g}$ & $\mathrm{q}$ & \\
\hline Africada & & & & $\left.d_{3},(t)\right)$ & & & \\
\hline Fricativa & $\mathrm{f}, \mathrm{v}$ & $\theta$ & $\mathrm{s}, \mathrm{z}$ & $\int, 3$ & $\mathrm{x}$ & & $\mathrm{h}$ \\
\hline Nasal & $\mathrm{m}$ & $\mathrm{n}$ & $\mathrm{n}$ & $\mathrm{n}$ & y & $\mathrm{N}$ & \\
\hline Aproximante & & & & $\mathrm{j}$ & $\mathrm{w}$ & & \\
\hline Lateral & & 1 & & & & & \\
\hline Vibrante & & & $\varsigma, \mathrm{r}$ & & & & \\
\hline
\end{tabular}

\begin{tabular}{|c|c|c|}
\hline & Anterior & Posterior \\
\hline Cerrada & $\mathrm{i}$ & \\
\cline { 1 - 1 } Intermedia & $\mathrm{e}$ & $\mathrm{o}$ \\
\hline Abierta & & $\mathrm{a}$ \\
\hline
\end{tabular}

Tabla 1. Inventario fonético del dothraki (Peterson 2015b: 92)

Como puede observarse en la tabla anterior, solo aparecen cuatro fonemas vocálicos: /a/, /e/, /i/ y /o/. El único elemento vocálico que no se utiliza es la /u/; tan solo se registra en el grupo qu- por iniciativa de Martin, como en Quaro y Jhiqui (nombres propios). Del mismo modo, destacan una serie de rasgos que conforman la esencia de este diseño: la fricativa velar sorda [x] (en khal 'jefe' o arakh 'espada curvada', símbolo dothraki), la vibrante múltiple [r] (en khalasar 'horda dothraki' o lajasar 'ejército'), la oclusiva uvular sorda [q] (en qoy 'sangre' o qora 'brazo'), la fricativa glotal [h] (que con frecuencia aparece como 
[ћ]) (en hrazef 'caballo' o mahrazh 'hombre') y las consonantes dobles y geminadas (en iffi 'victoria' o allayafat 'complacer'). El autor de la lengua se asegura de que estos sonidos aparezcan asiduamente en su lengua mediante el uso de una serie de estrategias, entre las que destaca la adición de afijos. Por ejemplo, Peterson convierte la fricativa velar sorda [x], representada como $k h$, en el sufijo derivativo -i(kh), que da lugar a términos como kachrakh 'oler', nesikh 'conocimiento', sewafikh 'vino', etc. (Gándara 2018: 320).

Este diseño lingüístico se ampara en los criterios de la simplicidad y la regularidad, por lo que tampoco presenta una morfología excesivamente compleja. En resumen, su sistema pronominal distingue entre singular y plural en las tres personas, a excepción de la segunda en la que aparece la forma yer 'tú' para un tratamiento familiar y shafka 'usted' o 'ustedes' para el formal ${ }^{6}$. Los verbos pueden ser de dos tipos en función de si terminan en vocal (a los que se añade -lat para formar el infinitivo) o en consonante (a los que se suma -at). Su paradigma verbal no presenta una gran complejidad, con diferentes terminaciones en función de la persona y el tiempo. Para expresar la negación, se utilizan las partículas vos o vo en posición preverbal para los terminados en vocal o consonante, respectivamente. El significado de ser se expresa mediante la unión de un sustantivo en caso nominativo y otro término; en suma, una oración como 'El hombre es un guerrero" se traduce como Mahrazh lajak. El verbo tener, por su parte, se formula con la expresión invariable mra qora (literalmente 'en la mano'), que debe estar siempre acompañada de un nombre en caso nominativo, como en Arakh mr qora o 'una espada está en mi mano/tengo una espada'.

El dothraki presenta un sistema de género basado en las categorías de animado e inanimado en los nombres ${ }^{7}$. Existe así una correlación importante entre género gramatical y tipo de entidad: los seres vivos se consideran animados y los inertes, inanimados. Estos no son fáciles de identificar en dothraki, pues entes inanimados en la cultura occidental, como río o cabeza, son animados en esta lengua. Al contrario, también cabe destacar que términos como hrazef 'caballo' pertenecen a los inanimados en dothraki. Este hecho es relevante si tenemos en cuenta la importancia que tiene este animal en su cultura. Como animados, destacamos rizh 'hijo', ashefa 'río' o nhare 'cabeza'. Por otro lado, nombres inanimados son rhiko 'estribos', zhavvrosa 'dragón' o hlofa 'muñeca'. Peterson toma como referencia la importancia que tienen los seres en la cultura dothraki para asignarles una u otra clase. Dicha distinción es también relevante para la categoría morfológica del número, pues solo los nombres animados admiten marca de plural. Además, todos los vocablos construidos con sufijos de colectividad (-asar o -isir) constituyen entes animados, como hoyalasar 'música' y ikhisir 'cenizas'.

Desde el punto de vista de su clasificación, el dothraki es una lengua flexiva que posee un sistema de cinco casos: nominativo ( $\operatorname{rrakh}$ 'espada'); acusativo ( $\operatorname{rrakh}$ 'espada); genitivo

6 Los pronombres personales son los siguientes: Anha 'yo'; yer 'tú'; shafka 'usted' o 'ustedes'; me 'él' o 'ella'; kisha 'nosotros'; yeri 'vosotros'; mori 'ellos'.

7 La oposición entre animado/inanimado para establecer el género gramatical de las palabras fue esencial en el indoeuropeo común. Sin embargo, en su desarrollo posterior tendió a desaparecer, salvo en las lenguas eslavas (ruso, polaco, checo, etc.) que continúan manteniendo dicha distinción e incluso establecen diferenciaciones dentro de la misma (Violi 1991: 38). No obstante, en el caso concreto del dothraki, es posible que Peterson tomara como referencia las lenguas algonquinas, distribuidas geográficamente en el noreste y centro de América. Aunque su sistema de género no se corresponde con el del dothraki, estas clasifican los nombres en animados (seres con vida y algunos objetos tradicionales con poderes espirituales) e inanimados (seres inertes). 
(arakhi 'de la espada'); alativo (arakhaan 'a la espada') ; y ablativo (arakhoon 'de la espada'). A excepción del alativo, que aparece en las lenguas ugrofinesas y bálticas, el resto de casos está presente en latín. El orden de palabras es Sujeto Verbo Objeto (SVO), como en khalaka dothrae mr anhal, cuya traducción es 'Un príncipe cabalga en mí'.

La información sobre la gramática de esta lengua inventada se recoge en The art of language invention. From Horse-Lords to Dark Elves, the Words Behind World-Building (Peterson 2015) y en el manual Living Language Dothraki (Peterson 2014). En este último, se incluye un glosario de términos en dothraki que nos han permitido trazar el estudio del léxico que presentamos a continuación.

\section{LA CREACIÓN LÉXICA EN CANCIÓN DE HIELO Y FUEGO Y SU INFLUEN- CIA EN LA CONSTRUCCIÓN DE LA LENGUA DOTHRAKI}

En primer lugar, para trazar un estudio del léxico del dothraki conviene diferenciar entre aquellos términos creados por George R. R. Martin para las novelas y los construidos por David J. Peterson para la serie. Los primeros determinan decisivamente a los segundos, pues Peterson se basa en las palabras que Martin había incorporado a sus novelas para construir su sistema lingüístico. Son las siguientes ${ }^{9}$ :

\begin{tabular}{ccccc}
\hline \multicolumn{5}{c}{ PALABRAS EN DOTHRAKI } \\
\hline khal & hrakkar & hranna & Cohollo & Ogo \\
khaleen & Jhogo & dosh & Rakharo & Temmo \\
rakh & Jommo & andahli & haesh & ko \\
dothrae & jaqqa & vaes & Aggo & Rhogoro \\
Dothraki & khalasar & Haggo & Iggo & quiya \\
Qotho & khas & Rhaego & Zollo & shierak \\
Fogo & rhaesh & Jhiqui & Bharbo & Moro \\
tolorro & khalakka & Mago & Pono & ai \\
khaeleesi & Drogo & rhae & maegi & Jhaqo \\
arakh & Quaro & mar & qoy & dothra \\
haj & Irri & rhaggat & rakhi & rham \\
& & & & mr'anha \\
\hline
\end{tabular}

Tabla 2. Términos inventados por George R. R. Martin

El léxico de este diseño lingüístico artificial está estrechamente relacionado con la cultura y las costumbres del pueblo dothraki. En consecuencia, como se observa en la tabla anterior, Martin elaboró todo un campo semántico para su organización jerárquica: de la raíz

8 El caso alativo (del latín allatus "llevado" + el sufijo español -ivo) aparece en las lenguas bálticas y finougrias. Se emplea para enunciar la dirección hacia donde se produce el movimiento. En dothraki, el caso alativo puede funcionar también como objeto indirecto, receptor u objetivo cuando acompaña a determinados verbos. El alativo con valor locativo aparece también en el quenya o alto élfico de J. R. R. Tolkien, en la que se traduce anteponiendo las preposiciones "a", "hacia" o "para" (González 2002: 90). Por lo tanto, Peterson podría haber tomado como referencia esta lengua artificial para establecer el sistema de casos del dothraki.

9 Las palabras que comienzan por mayúsculas son nombres propios. Nótese la diferencia con el resto. 
khal 'jefe de los dothraki' ${ }^{10}$ derivan khaleesi 'mujer del khal'; Khalakka 'príncipe, hijo del khal'; khalasar 'una horda fiel a un solo khal'; khasar 'un pequeño grupo de protectores o generales'; y khas 'forma corta de khasar'. Partiendo de este mismo lexema, Peterson construye vocablos relacionados con este campo semántico como khalakki 'princesa, hija del khal' mediante la adición del sufijo de género femenino -i y khaleessiya 'sirvienta', tomando como base la palabra khaleesi, aunque doblando la última $s$ y añadiendo -iya. Este último se convierte en un sufijo para indicar posesión, que también aparece en otros términos como jerriya 'debate, discusión' (de jerak 'comerciante') en el que se dobla la consonante de la sílaba anterior. Lo mismo sucede con marriya 'herramienta', que deriva del verbo en infinitivo marilat 'construir', cuya desinencia en -lat se pierde en la conjugación del verbo; por este motivo, se produce la duplicación de la consonante $r$ de la última sílaba. De la misma forma, Peterson también utiliza el sufijo -eesi para indicar la profesión en femenino en el caso de koalakeesi 'curandera' frente a koalak 'curandero'"1. Estas últimas pertenecen también al campo léxico de la medicina:

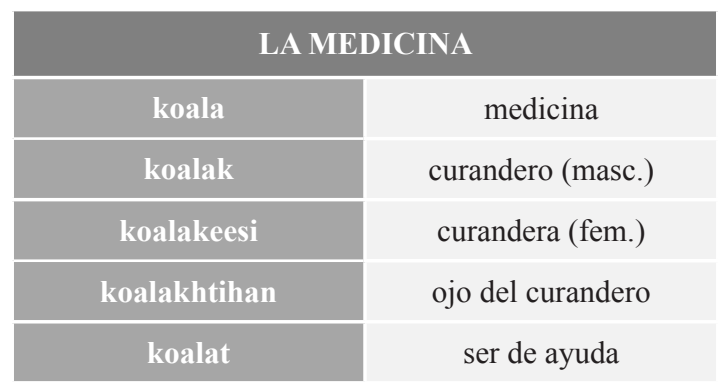

Tabla 3. Campo léxico de la medicina

En este ejemplo, conviene destacar también cómo el término koalat está formado por el sufijo para crear el infinitivo, mientras que koalakhtihan es una forma aglutinante que presenta la raíz koala $+(\mathrm{kh})+($ thin $)+($ an $)$. Thin significa 'ojo' y -an se presenta como sufijo para expresar posesión; este último aparece también en la forma quemmotihan 'párpado'. Por otra parte, es posible observar cómo Peterson convierte el término ko, cuyo significado es 'guardaespaldas' en una raíz a la que añade afijos para formar palabras que, salvo excepciones, no guardan relación semántica entre sí. De este modo, mientras observamos ejemplos como koge 'corte' o 'defecto' y kogmen 'perfecto' (adjetivo) que sí están vinculadas, encontramos otros como kohol 'arco' y kovarat 'reposar' o kolver 'águila' y kolverikh 'golpe con la espada recta' que comparten la misma raíz pero no tienen relación en su significado.

Martin también crea otros vocablos como rakh 'niño cordero'; rhae 'pie, pierna'; rhae mhar 'pie lastimado'; rhaesh 'tierra, campo' y rhaggat 'carro'. En esta misma línea, Peterson convierte rhae en una raíz a la que añadirle sufijos para designar conceptos que no siempre se encuentran relacionados por su significado, como rhaek 'semental'; rhaesheser 'mundo'; rhaesof 'zancada, yarda'; e incluso formas compuestas como rhaggat eveth 'bar-

10 En la pronunciación se encuentran diferentes variantes, entre ellas khal y kal.

11 El sufijo -essi puede ser una deformación del inglés, como en stewardess 'azafata' / steward 'azafato'. 
co' y rhaesof Valiri 'metro'. Además, Martin también construye otras palabras sin aparente relación formal pero referentes a aspectos importantes en la cultura dothraki. Algunas de estas son: tolorro 'hueso'; jaqqa 'verdugo'; dosh 'consejo'; vaes 'ciudad'; qoy 'sangre' haesh 'hueva'; shierak 'estrella'; y ai 'sí'; entre otras. Finalmente, es posible encontrar un total de 24 nombres propios acabados mayoritariamente en -o, a excepción de Jhiqui e Irri (véase Tabla 1). En conclusión, conviene destacar que estas creaciones léxicas no presentan una sistematicidad evidente en su mayoría, sino que Martin simplemente pretende otorgar una mayor verosimilitud y originalidad a uno de los pueblos que habita en el universo imaginario de Canción de hielo y fuego.

\subsection{El diseño del léxico por David J. Peterson}

El listado de palabras creado por Martin en las novelas fue decisivo para la configuración del léxico de la lengua dothraki. Peterson tomó como modelo las creaciones lingüísticas del autor de la saga literaria para establecer el siguiente esquema ${ }^{12}$ :

\begin{tabular}{|c|c|c|}
\hline BOOK WORD(S) & PATTERN(S) & DERIVED COINAGES \\
\hline $\begin{array}{l}\text { tolorro "bone(s)" } \\
\text { khalakka "prince" }\end{array}$ & CVCVCCV & $\begin{array}{l}\text { jorokkeya 'corn husk', Kovarro } \\
\text { 'man's name', varanna '(s/he) will be } \\
\text { neglected' }\end{array}$ \\
\hline arakh "sword" & (C) $\mathrm{VCVC}$ & $\begin{array}{l}\text { eyel 'rain', jalan 'moon', oyof 'clay', } \\
\text { qazat 'nine', tokik 'fool' zajikh } \\
\text { 'refusal' }\end{array}$ \\
\hline hranna "grass" & $\begin{array}{l}h r-, h r-, h \\
C[+ \text { liquid }]\end{array}$ & $\begin{array}{l}\text { hrazef 'horse', hrelki 'mushroom', } \\
\text { holfa 'wrist', mahrazh 'man' }\end{array}$ \\
\hline $\begin{array}{l}\text { mhar "sore" } \\
\text { anha "I" }\end{array}$ & $\begin{array}{l}m h-, m h, n h \\
\mathrm{C}[+ \text { nasal }] h\end{array}$ & $\begin{array}{l}\text { mhotha 'barley', mhegga 'dried } \\
\text { dung', nhare 'head', janha 'blanket' }\end{array}$ \\
\hline $\begin{array}{l}\text { rhae "foot" } \\
\text { dothrae "rides" }\end{array}$ & $a e, \mathrm{VV}$ & $\begin{array}{l}\text { aena 'dawn', lahklae 's/he pants', } \\
\text { lathe '( } \mathrm{s} / \mathrm{he}) \text { wakes up', khaor 'waist' }\end{array}$ \\
\hline
\end{tabular}

Tabla 4. Estructura de las palabras inventadas en Canción de hielo y fuego (Peterson, 2015b: 26)

En la tabla anterior, se observa cómo Peterson utiliza la estructura silábica de los vocablos de Martin para establecer unos patrones con los que elaborar nuevos términos. No obstante, en el diseño del dothraki influyeron otros factores externos al acto de creación lingüística. El hecho de construir una lengua para el cine obligó a Peterson a cumplir unos plazos establecidos y, en algunas ocasiones, a agilizar el proceso de invención de su pro-

12 La importancia que Peterson otorga a la forma y combinación de los vocablos se observa en la siguiente cita: "Word shape, I felt, was crucial in making the extant Dothraki vocabulary feel as if it had sprung from the larger work. For example, given the phonotactics of English -the particular combinations of sounds that the language allows -kanibunaki, angmeeloo, haborbori are all licit potential words, because they follow English syllable patterns, but hey simply do not look like English words, especially when compared to other licit examples like cring, runk, dilk and hean. A language will often admit a much wider variety of potential word shapes than it ultimately makes use of" (Peterson, 2015b: 26). 
yecto. Con este fin, el lingüista elaboró de manera anticipada un gran número de términos que le facilitaron enormemente el proceso de traducción al dothraki. De este modo, destaca el autor que "while a conlang of mine like Kamakawi had accrued just over 2.000 words in the eight years I'd been working on it, Dothraki had over 1.700 words after the initial two month application process" (Peterson 2015b: 28).

Para la configuración del vocabulario, Peterson tomó como modelo el diccionario que ya había creado para otra de sus lenguas, el zhyle, un sistema basado en la suma de sufijos a una raíz determinada. El procedimiento de creación del dothraki fue similar al de esta lengua. La construcción de nombres, adjetivos y verbos se efectuó de manera sistemática, partiendo de una serie de raíces a las que agregó diferentes afijos, aunque con algunas irregularidades. Pues, como señala el autor, "building up the language in this fashion was much more efficient than the word-by-word construction process I utilized with Kamakawi" (2015b: 28). Dicha sistematicidad se observa en los ejemplos del siguiente cuadro, en el que se establece una comparación entre ambas lenguas:

\begin{tabular}{lll} 
LANGUAGE & ROOT & \multicolumn{1}{c}{ DERIVED WORDS } \\
\hline Zhyler & mat & $\begin{array}{l}\text { matal 'to see', madga 'eye', matler 'horizon' matty 'judge' matsha } \\
\text { 'glasses', madja 'telescope' mattha 'wisdom' }\end{array}$ \\
Dothraki & tih & $\begin{array}{l}\text { tih 'eye', tihat 'to see', tihilat 'to look at', tihak 'scout', tiholat 'to } \\
\text { understand', tihikh 'view', tihi 'glance', attihat 'to show' }\end{array}$
\end{tabular}

Tabla 5. Un ejemplo de derivación en zhyler y en dothraki (Peterson, 2015b: 28)

Por otro lado, el objetivo de Peterson fue siempre crear una lengua cuyo funcionamiento fuese idéntico al de las lenguas naturales. Por este motivo, tiende a reutilizar palabras ya inventadas, como afirma en las siguientes líneas:

Though one thinks of a conlanger as someone who creates words, creating a brand new word is always my last resort. If a new word is needed, I always ask myself this question first: What have I already got? Recycle, reduce, reuse, This is what we do with our own languages, so it stands to reason that if one's conlang is supposed to look like one of our languages, one should do the same (Peterson 2015a: 184).

Construir una lengua con un funcionamiento similar a una lengua natural resulta una tarea complicada si se trata de un sistema diseñado para el cine. No solo porque sea necesario poseer un amplio caudal léxico para satisfacer las necesidades que surgen en las grabaciones de la serie, sino también porque un conlanger no siempre puede estar presente en los rodajes $^{13}$. Este hecho puede implicar una falta de comunicación entre conlangers y productores, algo que provoca errores como el que Peterson expone en esta cita:

13 Conlanger es el término empleado en inglés para referirse a un inventor de lenguas. Proviene del término "conlang", suma de la primera sílaba de los vocablos ingleses "constructed" y "language”. Aparece por primera vez el 29 de julio de 1991 para denominar la lista de correo electrónico en la que se debatían cuestiones relacionadas con la creación de lenguas. Esta palabra ha sido aceptada por el Oxford English Dictionary y el Cambridge Dictionary. 
For example, in episode 1.03 of Games of Thrones ("Lord Snow"), there's a line I completely mistranslated. I got an e-mail that simply asked for the translation of a new line for Jorah: "Stop the Horde!" Without context, I really didn't know what was intended, so I translated it as Annakhas dozgosores! Annakhas is the command form of annakhat, which means "to cause/force to stop". Dozgosores is the accusative of dozgosor, a collective of the word dozgo which means "enemy". Unfortunately, Jorah wasn't giving a command to stop the onslaught of an oncoming enemy horde: He was telling someone else to cause the Dothraki khalasar they were riding in to halt so that the khaleesi could get off her horse for a bit. As a result, I had to find a way to fold dozgosor into the vocabulary as a superlative term for one's own people, in addition to a word for an enemy -a not impossible, but nevertheless unpalatable task (Peterson 2015a: 29-30).

En suma, para construir el vocabulario de la lengua dothraki, Peterson establece una serie de raíces base de las que derivan el resto de términos. En algunos casos, crea diferentes campos semánticos relacionados tanto en forma como en significado, aunque dentro de estos es posible encontrar ciertas irregularidades. Para la formación de palabras, el conlanger emplea tanto procedimientos propios de la composición como de la derivación, aunque es evidente la supremacía de esta última, como veremos más adelante. Por tanto, en las siguientes páginas de este estudio repasaremos, primeramente, algunos de los campos semánticos y léxicos más importantes de la lengua dothraki, con el fin de destacar las similitudes y diferencias que se evidencian entre ellos, para observar, en segundo lugar, los principales procedimientos de formación morfológica empleados.

\section{CORPUS LÉXICO}

En primer lugar, en este apartado exponemos algunos de los campos léxicos y semánticos más importantes de la lengua dothraki. Incluimos un análisis de algunos de las asociaciones que se establecen entre los vocablos que conforman dichos campos.

\subsection{La familia}

La primera de las tablas que presentamos establece los diferentes términos creados por Peterson para el campo semántico de la familia ${ }^{14}$ :

\begin{tabular}{|l|l|l|}
\hline \multicolumn{2}{c|}{ LA FAMILIA } \\
\hline rhojosor (n.a.) & family & familia \\
\hline ave (n.a.) & father & padre \\
\hline mai (n.a.) & mother & madre \\
\hline rizh (n.a.) & son & hijo \\
\hline ohara (n.i.) & daughter & hija \\
\hline gaezo (n.a.) & brother & hermano \\
\hline inavva (n.a.) & sister & hermana \\
\hline simon (a.n) & uncle & tío \\
\hline krista (n.a.) & aunt & tía \\
\hline
\end{tabular}




\begin{tabular}{|l|l|l|}
\hline \multicolumn{2}{c}{ LA FAMILIA } \\
\hline mhrazh (n.a.) & husband & marido (forma corta de mahrazhkem) \\
\hline chiori (n.a.) & wife & mujer (forma corta de chiroikem) \\
\hline kemak (n.a.) & spouse & esposa \\
\hline yalli (n.i.) & child & niño \\
\hline simonof (n.i.) & grandfather & abuelo \\
\hline kristasof (n.i.) & mother who is still breast feeding & abuela \\
\hline drane (n.a.) & madre que todavía está amamantando \\
\hline siera (n.a.) & nephew & sobrino \\
\hline janise (n.a.) & $\begin{array}{l}\text { niece } \\
\text { orphan }\end{array}$ & sobrina \\
\hline leishak (n.a.) & ancestor & antepanado \\
\hline kim (n.a.) & & \\
\hline
\end{tabular}

Tabla 6. Términos inventados para la familia (Peterson 2014: 89-9) ${ }^{15}$

Como puede observarse en la tabla anterior, no hay regularidad alguna en la creación de palabras dentro de este campo semántico. Tan solo observamos algunas similitudes en la formas de estas creaciones léxicas. Resulta cuanto menos curioso que los únicos términos que comparten la misma raíz son simon 'tío' y krista 'tía' (también 'pariente femenino') que han derivado en simonof 'abuelo' y kristasof 'abuela' mediante la suma del sufijo -of a las dos formas anteriores. Además, estos dos últimos términos, junto a yalli 'niño' aparecen como nombres inanimados en contraste con el resto. Esta elección podría justificarse por la escasa importancia que estos tienen en la cultura dothraki frente al término kim 'antepasado' que se categoriza como nombre animado.

\subsection{La lucha}

En cuanto al campo semántico de la lucha, Peterson presenta los siguientes términos, entre los que encontramos diferencias tanto en su forma como en su significado:

\begin{tabular}{|c|c|c|}
\hline \multicolumn{3}{|c|}{ LA LUCHA } \\
\hline addrivat & to kill & matar \\
\hline araggat & to choke & ahogar \\
\hline assolat & to command & mandar \\
\hline atthasat & $\begin{array}{l}\text { to defeat (insulting), to make somonel } \\
\text { someonething fall }\end{array}$ & derrotar (insultando) \\
\hline azzafirolat & to enslave & esclavizar \\
\hline azzisat & to harm someone & dañar a alguien \\
\hline fakat & $\begin{array}{l}\text { to kick; to kick at (when followed by noun } \\
\text { in allative case) }\end{array}$ & $\begin{array}{l}\text { patear; para patear (cuando va seguido de } \\
\text { nombre en alativo) }\end{array}$ \\
\hline fatat & $\begin{array}{l}\text { to slap; to slap at (when followed by noun } \\
\text { in allative case) }\end{array}$ & $\begin{array}{l}\text { abofetear; dar una palmada en (cuando va } \\
\text { seguido de nombre en alativo) }\end{array}$ \\
\hline
\end{tabular}

15 La traducción de los términos al español es de la autora. 


\begin{tabular}{|c|c|c|}
\hline \multicolumn{3}{|c|}{ LA LUCHA } \\
\hline fatilat & $\begin{array}{l}\text { to insult; to throw an insult at (when } \\
\text { followed by noun in allative case) }\end{array}$ & $\begin{array}{l}\text { insultar; lanzar un insulto a (cuando va } \\
\text { seguido de nombre en alativo) }\end{array}$ \\
\hline kaffat & to crush & aplastar \\
\hline lajat & to fight & pelear \\
\hline lojat & $\begin{array}{l}\text { to hit; to hit at (when followed by noun in } \\
\text { allative case) }\end{array}$ & $\begin{array}{l}\text { golpear; golpear a (seguido de nombre en } \\
\text { alativo) }\end{array}$ \\
\hline najahat & to be victorious & ser victorioso \\
\hline ovvethat & to shoot (with a bow), to throw & disparar (con arco), lanzar \\
\hline qoralat & to seize, to hold & agarrar, sujetar \\
\hline saqoyalat & to be bloody & ser sangriento \\
\hline vijazerat & to protect & proteger \\
\hline vindelat & $\begin{array}{l}\text { to stab; to stab at (when followed by noun } \\
\text { in allative case) }\end{array}$ & $\begin{array}{l}\text { apuñalar; apuñalar al (con nombre en } \\
\text { alativo) }\end{array}$ \\
\hline zisat & to be hurt & estar herido \\
\hline
\end{tabular}

Tabla 7. Campo semántico de la lucha (Peterson 2015a: 92)

Por otra parte, dado que este es un pueblo guerrero, Peterson decidió crear numerosas palabras y expresiones relacionadas con este campo léxico. En primer lugar, exponemos aquellos términos destinados a expresar "sufrimiento" y "dolor físico". Como puede observarse, no hay sistematicidad ni relación formal entre ellos, aunque sí es posible encontrar algunas formas derivadas o relacionadas con estas en el diccionario de Littauer (2016).

\begin{tabular}{|l|l|l|}
\hline \multicolumn{2}{|c|}{ SUFRIMIENTOS Y DOLORES EN EL CUERPO } \\
\hline annithat & to hurt & herir \\
\hline athnithar & pain & dolor \\
\hline athmharar & ache, soreness & dolor, inflamación \\
\hline mhari & headache & dolor de cabeza \\
\hline ziso & wound & herida \\
\hline quiya & bleeding & sangría \\
\hline
\end{tabular}

Tabla 8. Dolor y sufrimiento

El pueblo dothraki utiliza una amplia variedad de armas en la batalla; la más común es el arakh, una 'espada curvada', que sirve además de seña de identificación de este grupo en la serie. Otros nombres de armas son: orvik 'látigos', kohol 'arcos', laqam 'flechas', mihesof 'dagas o puñales' y gehqoyi 'boleadoras o bolas'. Por otro lado, los dothraki no usan armadura, ya que impide su velocidad y movimiento en la batalla. Casi siempre atacan a caballo, siendo muy pocas las veces que atacan a pie. Su valor reside en su fuerza. Todos los guerreros dothraki (lajaki) exhiben una trenza (jahak), que solo se cortan en caso de ser derrotados; este se considera un acto vergonzoso para un guerrero. Pese a su inclinación por la guerra, las espadas no pueden desenvainarse en la única ciudad dothraki, Vaes Dothrak, en la que está prohibido llevar armas. Finalmente, dado que son una cultura sangrienta, su lengua alberga varios verbos para el signi- 
ficado de 'matar'; entre los más comunes, se encuentran: 1) Addrivat: significa literalmente to make something dead y es usado cuando el asesino es un ser racional; 2) Drozhat: normalmente se utiliza cuando el causante de la muerte es un animal o un objeto inanimado. Con objetos inanimados se refiere a casos de muerte accidental. Drozhat se emplea también para describir una muerte fruto de una batalla descabellada, en la que el asesino ha actuado más como un animal que como una persona; 3) Ogat: es similar a sacrificar o matar a un animal (matanza).

\subsection{El cuerpo humano}

De igual forma, las palabras creadas para el campo semántico del cuerpo humano son las siguientes:

\begin{tabular}{|c|c|c|}
\hline \multicolumn{3}{|c|}{ EL CUERPO HUMANO } \\
\hline nhare (n.a.) & head & cabeza \\
\hline hatif (n.i.) & face & cara \\
\hline noreth (n.i.) & hair & pelo \\
\hline chare (n.i.) & ear & oreja \\
\hline tih (n.a.) & eye & ojo \\
\hline riv (n.i.) & nose & nariz \\
\hline gomma (n.a.) & mouth (of a person) & boca \\
\hline lekh (n.i.) & tongue & lengua \\
\hline qora (n.a.) & arm/hand & brazo/mano \\
\hline torga (n.a.) & stomach & estómago \\
\hline rhae (n.a.) & foot/leg & pie/pierna \\
\hline qoy (n.i.) & blood & sangre \\
\hline tolorro(n.i.)(acusativo: tolor) & bone & hueso \\
\hline kher (n.i.) & flesh & carne \\
\hline meso (n.i.) & muscle & músculo \\
\hline ilek (n.i.) & skin & piel \\
\hline zhor (n.a.) & heart & corazón \\
\hline
\end{tabular}

Tabla 9. El cuerpo humano

En este caso, es interesante observar, primeramente, la diferencia entre nombres animados e inanimados dentro de este campo semántico, aunque Peterson no aporta explicación alguna sobre esta distinción. Además, resulta interesante advertir cómo el autor engloba en un mismo vocablo los significados para 'pie'/'pierna' en rhae y 'mano'/'brazo' en qora, tal y como sucede en otras lenguas como el japonés.

\subsection{La comida}

En el campo semántico de la comida no encontramos relación formal entre los términos, a excepción de nindi 'salchicha' y ninthqoyi 'morcilla' y zhif'sal' y zhifikh 'carne salada'. En 
el primer caso, nindi se une a qoyi en caso genitivo, lo que provoca que se pierda la oclusiva dental /d/ y la vocal /-i/ final para dar lugar al dígrafo th. A continuación, exponemos en la siguiente tabla otros conceptos pertenecientes a este campo semántico:

\begin{tabular}{|l|l|l|}
\hline \multicolumn{3}{c}{ LA COMIDA } \\
\hline hadaen & food & comida \\
\hline gavat & dry, salted meat & carne \\
\hline zhifikh & duck & pato \\
\hline alegra & lamb & cordero \\
\hline vafi & pork & cerdo \\
\hline qifo & sausage & salchicha \\
\hline nindi & blood sausage & morcilla \\
\hline ninthqoyi & salt & sal \\
\hline zhif & honey & miel \\
\hline givikh & soup & sopa \\
\hline Mesina & stew & estofado \\
\hline lashfak & feast & banquete \\
\hline vitteya & & \\
\hline
\end{tabular}

Tabla 10. Campo semántico de la comida (Peterson 2014: 96)

Como puede apreciarse, no hay apenas relación entre los términos de este campo semántico. De tal forma, para el acto de 'comer' se utiliza adakhat, del que deriva adakhilat 'alimentar' o 'alimentarse' (también 'darse un festín'). Sin embargo, para 'alimentar' también se emplea el término vadakherat, formado mediante la adición de la consonante v- al inicio de la palabra y el sufijo -erat al final. El término usado para 'beber' es indelat mientras que para 'cocinar' se recurre a jolinat. El primero no guarda relación semántica con ningún otro vocablo; sin embargo, de la raíz del segundo (jolin) derivan jolinikh 'comida preparada' y jolino 'cacerola'. Por otro lado, relacionados con la comida y la bebida, se registran dos verbos, garvolat y fevelat, cuyo significado varía en función de si su forma es intransitiva o transitiva. Lo vemos en la siguiente tabla:

\begin{tabular}{|lll} 
VERBO & INTRANSITIVO & TRANSITIVO \\
\hline garvolat & lit. crecer (to grow hungry) & $\begin{array}{l}\text { tener hambre de (to hunger for) (cuando es seguido por un } \\
\text { nombre en ablativo) }\end{array}$ \\
\hline fevelat & lit. sed (to thirst) & $\begin{array}{l}\text { ansias de (to thirst for) (cuando va seguido por un nombre } \\
\text { en ablativo) }\end{array}$
\end{tabular}

Tabla 11. Garvolat vs. fevelat

Finalmente, también relacionado con este campo léxico, encontramos el término lekhilat, que se traduce como 'probar'. Esta forma procede de lekhi, cuyo significado es 
'gusto' (en alemán, Leker) y da origen a otras como lanlekhi ('darse un banquete por puro placer') mediante la suma del prefijo -lan. No obstante, para entender la terminología relacionada con la alimentación en dothraki, cabe destacar que este pueblo se alimenta a base de carne de caballo, a la que denominan gavat o zhifikh, si esta se seca con sal, o lamekh si se fermenta con leche de yegua. En este sentido, las costumbres de los dothraki se encuentran en consonancia con el propio carácter de este pueblo; no solo porque tienden a comer carne sin cocinar, sino también porque obligan a las mujeres embarazadas a comerse el corazón crudo de un caballo tras habérselo arrancado al animal para que el feto crezca sano y fuerte. Por último, por mencionar algún aspecto más, esta lengua también tiene vocablos para los platos esenciales de los dothraki: las morcillas o ninthqoyi y la tarta de sangre o fosokhqoyi.

\subsection{La cultura del caballo}

La cultura dothraki gira en torno a la figura del caballo. Por ello, existe una gran cantidad de términos referentes a este campo léxico. En primer lugar, debemos hacer hincapié en la diferencia entre el caballo como animal, denominado hrazef, y el semental, vezh. Como hemos señalado en líneas anteriores, el primero se considera inanimado para marcar la diferencia con respecto al Gran Semental, que tiene una mayor importancia para el pueblo dothraki. No obstante, en cuanto al primero, Peterson escoge la raíz hra, utilizada también por Martin, para formar el término hrazef 'caballo' y algunos derivados como hrazef chafi 'caballo usado para domesticar' o hrazefeser 'manada de caballos salvajes'. Obsérvese la similitud semántica existente entre ambos términos. No obstante, dado que este es un pueblo nómada que se desplaza siempre a caballo, encontramos una amplia variedad de términos para referirse a los diferentes equinos: loal 'potro'; mare 'yegua'; jedda 'poni'; sajo 'corcel'; y manin 'yegua joven'. En algunas ocasiones, también encontramos casos derivados de estos vocablos, como en sajo, que deriva en sajak 'jinete' o sajat 'montar a caballo'. Hay también múltiples términos para diferenciar por colores a los diferentes equinos: nozho 'castaño'; cheyao 'alazán'; ocha 'pardo'; qahlan 'palomino'; y messhih 'perlino'. En este mismo ámbito, se registran algunas palabras referentes a la equitación: javrath 'riendas'; darif 'montura'; y rhiko 'estribos'. Destacan, además, varios nombres para las distintas formas de 'cabalgar': karlinat 'galopar'; karlinat + nombre en genitivo 'galopar junto a'; chetirat 'a medio galope'; chetirat + nombre en genitivo 'a medio galope junto a' (nótese cómo la terminación del nombre en genitivo modifica el significado del verbo en ambos casos); gorat 'cargar un caballo' y javrathat 'llevar las riendas' (derivado mediante sufijación verbal de javrath 'riendas'). En esta misma línea, encontramos algunas expresiones relacionadas con el mundo equino como: Soroh! ‘Alto!; Hosh! ‘iArre!’; Affa! ‘ßBasta!’; entre otras.

En este campo, el vocabulario relacionado con el término que da nombre a la lengua cobra especial importancia. Peterson utilizará el vocablo dothraki, que aparece ya en el primer libro de Canción de hielo y fuego, para generar una serie de palabras relacionadas con la cultura de este pueblo: dothrak 'jinete'; dothrakh 'viaje a caballo'; dothrakhqoyi 'jinete de sangre'; entre otros. Atendiendo a este mismo término, crea el verbo dothralat y le asigna tres significados distintos: 1) cabalgar (con caballo, corcel, o mi caballo, mi corcel como sujeto); 2) cabalgar junto a (verbo seguido de nombre en genitivo); 3 ) tener una erección. 


\subsection{Otros: la caza y la naturaleza}

El campo léxico de la caza se compone de los términos que aparecen en la siguiente tabla. Obsérvese cómo algunos de estos vocablos presentan una similitud en su forma, pero otros no se encuentran en absoluto relacionados. Destacamos también la gran cantidad de palabras existentes para referirse a las partes de un animal:

\begin{tabular}{|c|c|c|}
\hline \multicolumn{3}{|c|}{ LA CAZA } \\
\hline fonak & hunter & cazador \\
\hline fonakasar & hunting party & velada de caza \\
\hline idrik & leader of the hunt & líder de los cazadores \\
\hline fonat & to hunt & cazar \\
\hline drogat & to drive (animals) & transportar, manejar (animales) \\
\hline vadakherat & to feed & alimentar \\
\hline ovethat & fly & volar \\
\hline govat & to mate (animals), to breed & criar (animales) \\
\hline zorat & to roar & rugir \\
\hline ogat & to slaughter & masacrar \\
\hline ivezho & beast & bestia \\
\hline ivezh & wild & salvaje \\
\hline kadikh & captured animal, not yet tamed & animal capturado, aún no domesticado \\
\hline shim & tame & domesticar \\
\hline drogikh & herd & horda \\
\hline haesh & spawn & hueva \\
\hline oggo & head (of an animal) & cabeza (de un animal) \\
\hline hoska & mouth (of an animal) & hocico (de un animal) \\
\hline memzir & bird noise, "tweet" & pío \\
\hline felde & wing & ala \\
\hline feldekh & feather & emplumado \\
\hline eve & tail & cola \\
\hline chiva & horn & cuerno \\
\hline jahak & lion's mane & melena de un león \\
\hline hem & fur & pelaje \\
\hline hemikh & pelt & pellejo \\
\hline kher & skin & piel (de un animal) \\
\hline kherikh & leather & cuero \\
\hline dozgikh & animal carcass & esqueleto de un animal \\
\hline
\end{tabular}

Tabla 12. La caza 
En cuanto a la naturaleza, destacan aquellos elementos importantes para la cultura dothraki. Además, en estos se establece una clara distinción entre animados e inanimados. Entre los primeros, se encuentran: sorfosor 'tierra'; vorsa 'fuego'; jalan 'moon'; shierak 'estrella'; ramasar 'llanura'; ashefa 'río'; feshith 'árbol'; entre otros. En contraposición, entre lo segundos, subrayamos: eveth 'agua'; shekh 'sol'; shekhikh 'luna'; havazh 'mar'; krazaaj 'montaña'; hranna 'hierba'; etc. Como señalamos en líneas anteriores, dicha distinción se establece basándose en la importancia que tienen estos elementos en su cultura.

\section{PROCEDIMIENTOS DE CREACIÓN DE TÉRMINOS LÉXICOS}

Para formar las palabras de su lengua, Peterson toma como base una serie de raíces, con un determinado valor semántico, a las que añade diferentes afijos para precisar su significado. Este autor utiliza algunos de los mecanismos más socorridos para inventar nuevos términos, tales como la derivación y la composición. Con el fin de otorgar a su proyecto lingüístico artificial la apariencia de una lengua real en un contexto de ficción, el conlanger también emplea los acortamientos, las relaciones léxicas y los préstamos de otras lenguas. Hagamos un repaso por ellos:

\subsection{Derivación}

La creación de términos mediante sufijos es un mecanismo muy productivo en la lengua de Peterson. Estos le permiten crear familias de palabras con relación semántica y formal entre los términos. Por ejemplo, a partir del término lajak 'guerrero', el lingüista construye lajasar 'ejército' (con el sufijo de colectividad -asar); lajat 'luchar' (mediante el sufijo verbal -at); y lajilat 'entrenar o jugar (el juego se introduce con la preposición ki)' (con el sufijo verbal -lat). Vezh 'caballo semental', por su parte, da lugar a vezhak 'señor del caballo' a través de la desinencia -ak. Por último, también se añade -ven para constituir el adjetivo vezhven 'grande'. Otro ejemplo en el que se observa cierta sistematicidad es el siguiente: jesh 'hielo'; jesho 'helado'; jesholat 'helar'; jeshoy 'helado o congelado'; jeshven 'cubierto de hielo'. En el caso de kem 'casados', la suma de diversos sufijos origina los siguientes términos: kemak 'esposa'; kemik 'aliado'; kemisolat 'aliarse con alguien' (se usa con la preposición ma.); kemolat 'casarse' (utilizado con $m a$ ). En este último ejemplo, se establece un paralelismo entre 'casarse' y 'aliarse', que, además, puede relacionarse con el propio argumento de la obra ${ }^{16}$. Asimismo, kemis significa 'higo maduro' y se asocia con la abundancia y la fertilidad por las semillas de su interior.

La sistematicidad en la formación de palabras mediante sufijos se evidencia, por ejemplo, en la desinencia -men. En este caso, observamos tres vocablos en los que dicho sufijo implica el valor semántico 'sin': jahakmen 'sin trenza' (proviene de jahak 'trenza'); kogmen 'sin defectos' (de kog 'defecto'); y nithmen 'sin dolor' (de nith 'dolor'). Encontramos también el sufijo -eya, empleado en algunas ocasiones para la formación de sustantivos: challeya 'alubia' cuando se utiliza para referirse a la comida (derivado de chal 'alubia' como uso

16 El matrimonio concertado entre Khal Drogo y Daenerys Targaryen se debe a la anhelada alianza del hermano de Daenerys, Viserys Targaryen, con los dothraki para conquistar el Trono de Hierro. Este puede ser uno de los motivos por los que Peterson establece relaciones formales entre los términos kemisolat 'aliarse con alguien' y kemolat 'casarse'. 
general); najaheya 'victoria' (de najah 'victorioso'); vitteya 'festín' (deriva en vittheyqoyi 'día festivo'); entre otras. Asimismo, muestra de esta regularidad son los sufijos de colectividad -asar, -isir y -osor. Algunos ejemplos son: fonakasar 'velada de caza' > fonak 'cazador'; lajak 'guerrero' > lajasar 'ejército'17; rhaesh 'tierra o campo'> rhaesheser 'mundo'; hrazef 'caballo' > hrazefeser 'manada de caballos salvajes'; dozgo 'enemigo' > dozgosor 'horda enemiga'; sorfosor 'tierra' (no se documenta palabra relacionada); rhojosor 'familia' (no se registra relación con otro término); entre otros.

La productividad de los sufijos se muestra en el uso de las desinencias verbales -at y -lat, respectivamente. Además, la adición de una u otra terminación determina el significado del verbo: adakhat 'comer' y adakhilat 'alimentarse de'; charat 'oír' o 'enterarse de' y charolat 'escuchar'; jadat 'venir' y jadilat 'aproximarse'; qovat 'estremecerse' y qovvolat 'mudar la piel'; shillat 'confiar en' y shillolat 'creer'; entre otros. Como puede observarse en algunos de los ejemplos anteriores, la adición del sufijo verbal conlleva el desdoblamiento de la consonante anterior y, en algunos casos, la suma de una vocal epentética a la raíz cuando esta acaba en consonante. En aquellos términos en los que el lexema acaba en vocal, esta se pierde o se transforma con objeto de facilitar la formación mediante el sufijo verbal, como en ize 'veneno' $>$ izzat 'envenenar'. Finalmente, conviene destacar la construcción de vocablos mediante el sufijo -(i)kh, ideado por razones puramente fonéticas. Se observa en: dozgo 'enemigo'; 'para un enemigo específico'> dozgikh 'cadáver de un animal'; hem 'pelaje'> hemikh 'piel', ambos para animales; feld 'ala'> feldekh 'con plumas; tir 'dedo'; tirikh 'uña'; entre otros. En este caso, la palabra derivada mediante este sufijo no siempre guarda relación con la forma de la que proviene, como en hlizfil 'oso' y hlizifikh 'ataque de espada salvaje pero poderoso'.

En dothraki, aparecen también términos creados mediante prefijación. Al igual que sucede en las lenguas naturales, los prefijos se emplean con menor frecuencia en la creación de palabras. En el corpus de términos que conforman el léxico del dothraki (véase apartado 3), destacan los siguientes: lan-, presente en lanlekhi 'darse un banquete por puro placer', lanqoyi 'matanza'; os-, que aparece con valor de negación en ojil 'incorrecto', que proviene de jil 'correcto'; entre otros. Sin embargo, la escasez de términos formados exclusivamente mediante la prefijación contrasta con el gran número de palabras parasintéticas. Estas se utilizan con valores concretos, por ejemplo, para crear palabras abstractas de un término base; este es el caso de jahak 'trenza', de la que procede athjahakar 'orgullo', de gran importancia en la cultura dothraki. Con este valor, hay múltiples ejemplos, entre los que destacamos: athzalar 'esperanza' (zalat 'esperar'); athfiezar 'amor' (fiez 'cuerda'); athdrivar 'muerte' (drivat 'estar muerto') athhilezar 'sexo' (hilelat 'practicar sexo'); entre otras. Finalmente, otros ejemplos de palabras parasintéticas son: azzafrok 'esclavista' cuya raíz es zafra 'esclavo'; saqoyalat 'ser sangriento'; zichome 'irrespetuoso' (chomak 'respetuoso', insulto cuando se refiere a un dothraki).

\subsection{Composición}

En el marco de las lenguas construidas, los autores suelen acudir también a la composición como procedimiento para inventar nuevos términos. En el caso de Peterson, la mayoría de los nombres registrados se basan en la unión de dos raíces con significado

17 Entre las palabras creadas por Martin, se encuentra el sufijo -asar en la forma khalasar, construido a partir de khal. Por tanto, este es uno de los principios que Peterson hereda de Martin y que emplea con frecuencia en la construcción del dothraki. 
propio, como en lekhmove 'lengua construida': lekh 'lengua'18 + movelat 'hacer con las manos, crear'; yothnare 'cerebro' (de una persona): yoth 'fruta' + nhare 'cabeza' (de un humano); etc. El término qoy 'sangre' se utiliza para construir numerosas palabras en dothraki. Veamos un ejemplo:

\section{Qoy 'sangre'}

$$
\begin{gathered}
\text { fasqoyi 'destino' } \\
\text { fas 'nube' + qoy en genitivo 'de sangre' }
\end{gathered}
$$

karlinqoyi (lit. 'galopar lo suficientemente rápido para matar al caballo por agotamiento')

karlinat 'galopar' + qoy en genitivo 'de sangre'

lanqoyi 'matanza rápida'

lanat 'correr' + qoy en genitivo 'de sangre'

ninthqoyi 'morcilla'

nindi 'salchicha' + qoy en genitivo 'de sangre'

vorsqoyi 'pira funeraria'

vorsa 'fuego' + qoy en genitivo 'de sangre'

Tabla 13. Qoy 'sangre'

En dothraki, encontramos también formas compuestas mediante dos radicales simples con significado propio. Entre ellas, distinguimos algunos ejemplos como:

a. jerak sewafikhaan 'mercader de vino' > jerak 'mercader' + sewafikh 'vino';

b. lamekh ohazho 'leche de yegua fermentada' > lamekh 'leche de yegua' (derivada de lame 'yegua') + ohazho 'fermentado';

c. shierak quiya 'cometa' > shierak 'estrella' + qiya 'sangrante';

d. shor tawakof 'armadura' > shor 'vestido' + tawak 'metal';

f. rhae mhar 'dolor en el pie'> rhae 'pie' + mhar 'doloroso';

e. qoy qoyi 'sangre de mi sangre'> qoy en nominativo + qoyi (genitivo).

En algunos casos, Peterson utiliza la composición para la creación de nombres propios. Algunos de estos ya fueron establecidos por Martin en las novelas, tales como Dosh Khaleen 'consejo de viudas'; Haesh Rakhi 'los hombres corderos, Lhazareen'; Rhaesh Andahli 'Tierra de los Ándalos, Poniente'; Vaes Dothrak 'Ciudad Dothraki'; Vaes Seris 'Ciudades Libres'; y Vaes Tolorro 'Ciudad los Huesos'. En esta misma línea, Peterson construye los siguientes términos: Emrakh Hrazef 'Puerta del Caballo'; Havazh Dothraki 'Mar Dothraki'; Havazzhifi Khazga 'Mar de Sal Negra'; Hoyali Jeshi ma Vorsasi 'Canción de Hielo y Fuego'; Jeser Jim 'Mercado Occidental'; Jeser Tith 'Mercado Oriental'; Rhaeshi Ajjalani 'Tierras Sombrías'; Vilajerosh Adori 'Juego de tronos'.

18 En este caso, el término lekh se corresponde con el nombre animado 'idioma' o 'lengua', pues esta palabra, con idéntica forma pero con valor inanimado, significa 'parte del cuerpo'. 


\subsection{Acortamientos}

Generalmente, preposiciones y conjunciones suelen acortarse cuando la siguiente palabra comienza por una vocal. Así, es posible encontrar formas, tales como $h a>h$ ' 'de' o ' $\mathrm{a}$ '19; $k i>k$ ' 'por, a causa de' con genitivo, 'así, que' cuando precede al discurso citado; $m a>m$ ' 'y', con ablativo 'con'. En esta misma línea, aparecen también acortados algunos verbos auxiliares, como eth $>$ 'th (verbo aux. 'deber' o 'tener que', equivale a must, have to). Del mismo modo, es frecuente observar acortamientos en otros términos en dothraki, tales como akkovaras > akko! 'levántate' (uso específico para animales); m'ach o m'ath $>$ m'athchomaroon 'con respeto'; mahrazh > mahrazhkem 'marido'; entre otros.

\subsection{Relaciones léxicas}

En algunas ocasiones, Peterson no construye nuevas palabras, sino que cambia el significado de las que ya existen para formar nuevos términos. Para ello, recurre a la metáfora como mecanismo para establecer ciertas semejanzas entre dos o más conceptos. En dothraki, los conceptos metafóricos pueden aparecer organizados jerárquicamente. Es decir, la palabra para 'cabeza', nhare, se emplea también para referirse a la parte frondosa de un árbol; lenta 'cuello', para el 'tronco del árbol'; fotha 'garganta' para la parte interna el árbol; gadim, 'pulmones', originó gadima, la palabra que se utiliza para las raíces subterráneas del árbol. Por tanto, el resultado es una serie de términos interconectados que describe un sistema de conceptos relacionados. Por otro lado, también es posible encontrar vocablos formados mediante la metonimia. En este caso, destacan, por ejemplo, los términos rhae, que engloba los conceptos 'pie' y 'pierna', y qora, creado para 'mano' y 'brazo'.

\subsection{Préstamos de las lenguas valyrias}

Como en cualquier lengua natural, el dothraki presenta préstamos o extranjerismos propios de otras lenguas existentes en el universo fantástico de Juego de tronos. Este hecho permite a Peterson establecer relaciones entre sus lenguas inventadas, otorgándoles una mayor dosis de verosimilitud y autenticidad. Asimismo, al igual que en las lenguas naturales, las palabras tomadas de otra lengua sufren una adaptación en algunas ocasiones. Por ejemplo, un término importado del valyrio es havon 'pan'; en este caso, se conserva la misma forma. Sin embargo, no sucede lo mismo con el vocablo tembyr, perteneciente al alto valyrio, que se adapta al dothraki como timvir 'libro'. Finalmente, como nombres propios del valyrio, destacamos el término Dovoeddi 'inmaculado', que en valyrio de Astapor es Dovoghedhy. No obstante, Peterson también ideó una regla gramatical para los extranjerismos, ya que si estos no se declinan, deben aparecer siempre acompañados por la preposición haji.

19 La preposición ha puede utilizarse con ablativo y podría traducirse como 'de' y con alativo, cuya traducción posible sería 'a'. Si aparece acompañada del término nakhaan, se convierte en un adverbio, que significa 'por último, finalmente'. 


\section{CONCLUSIÓN}

El análisis del léxico del dothraki efectuado en las páginas precedentes nos ha permitido mostrar cómo se construye el vocabulario de una lengua artificial diseñada con propósitos artísticos. En este sentido, hemos observado, primeramente, cómo Peterson parte de las palabras que Martin incluye en Canción de hielo y fuego para construir nuevos términos en dothraki. Esto no siempre resulta fácil, ya que el autor de la saga literaria inventa casi aleatoriamente estas palabras para otorgar verosimilitud a su mundo de ficción. Peterson, por su parte, añade asistemáticamente sufijos a estos vocablos para designar significados distintos, como en el caso de rhae, que hemos analizado en páginas anteriores. En segundo lugar, hemos destacado cómo el conlanger presta especial interés en asociar su invención lingüística a la cultura que representará esta lengua. Para ello, crea diferentes campos léxicos y semánticos para los caballos, la comida, la familia, la violencia y la caza, entre otros. Esta anhelada simbiosis entre lengua y cultura se traduce en numerosas ocasiones en múltiples irregularidades tanto en la gramática como en el léxico de este diseño lingüístico. Sin embargo, estas anomalías no hacen desdeñable este proyecto, pues su estudio resulta interesante tanto por su singularidad (por su vinculación con una cultura guerrera - semejante a los Klingon- que idolatra a los equinos - con ciertas reminiscencias a la cultura mongola-) como por su alto grado de desarrollo. En su análisis, se advierte cómo Peterson posee un profundo conocimiento del funcionamiento de las lenguas, naturales o artificiales y, en cuanto al léxico, un dominio de los procedimientos de formación léxica empleados. Esto le ha permitido elaborar un proyecto con una gramática y un léxico amplios y cumplir así con su objetivo de idear un sistema lingüístico artificial con apariencia de lengua natural. Por tanto, este diseño - como tantos otros olvidados por considerarse meras curiosidades lingüísticas (Galán, 2018: 14)—, merece ocupar un lugar destacado en esa larga lista de proyectos que cimienta la historia del movimiento de creación de lenguas artificiales.

\section{REFERENCIAS BIBLIOGRÁFICAS}

Albani, Paolo y B. Buonarroti (2010). Dictionnaire des langues imaginaires. Paris: Les Belles Lettres. Balbuena Castro, A (2016). "De Hobbits, Tronos de Hierro y Vikingos. Desarrollo narrativo y cronológico de la fantasía épica", Tonos digital: Revista electrónica de estudios filológicos, 31: https://digitum.um.es/digitum/handle/10201/50283

Barnes, L. y C. van Heerden (2006). "Virtual languages in science fiction and fantasy literature", Language Matters,

37, 1, pp. 102-117.

Calero Vaquera, M. L. (1999). Proyectos de lengua universal. La contribución española. Córdoba: Publicaciones de la Universidad de Córdoba y Obra Social y Cultural Cajasur.

Calero Vaquera, M. L. (2010). "Las irregularidades lingüísticas desde la perspectiva de los inventores de lenguas universales". En Sinner, C. y A. Zamorano (eds.). La excepción en la gramática española. Perspectivas de análisis. Madrid y Frankfurt: Iberoamericana Editorial Vervuert.

Couturat, L. y L. Leau (1903). Histoire de la langue universelle. Hildesheim: Georg Olms Verlag.

Eco, U. (1994). La búsqueda de la lengua perfecta. Barcelona: Crítica.

Galán Rodríguez, C. (2012). "Lenguas artificiales”. En Zamorano, A. (ed./coord.). Reflexión lingüística en la España del XIX. Marcos, Panoramas y Nuevas Aportaciones. Munich: Lincom Europa, pp. 417-442. 
Galán Rodríguez, Carmen (2018). Glosolalias femeninas e invención de lenguas. Córdoba: Editorial Universidad de Córdoba.

Gándara Fernández, L. (2018). "Introducción a la fonética del dothraki, lengua de Juego de tronos", Estudios humanísticos. Filología, 74, 1, pp. 309-326.

Gándara Fernández, L. (2019). "Un análisis lingüístico de la lengua Klingon (Star Trek)”, Tonos digital: Revista de estudios filológicos, 37.

Grande Alija, F. J. (2008). "Diccionarios, lenguas perfectas y el nombre de las cosas", Boletín de Filología, XLIII, pp. 109-143.

González Baixauli, L. (2002). La lengua de los elfos. Una gramática para el quenya de J. R. R. Tolkien: Tengwesta Kwenyava. Barcelona: Minotauro: https://drive.google.com/file/d/1Dizb0Ynd U0MROsorTdYiuqtWXto3nxT/view (04-02-20).

Jordan, D. K. (1997). "Esperant and Esperantism: Symbols and Motivations in Movement for Linguistic Equality". En Tonkin Humphrey (ed.). Esperanto, Interlinguistics, and Planned Languages. Lanham, MD: University Press of America.

Knowlson, J. (1975). Universal Language Schemes in England and France, 1600-1800. Toronto: University of Toronto Press.

Littauer, R. (2016). “The Dothraki Language Dictionary": https://docs.dothraki.org/Dothraki.pdf (0202-18).

Martínez Gavilán, M. D. (2016). "La contribución de Caramuel a la creación de lenguas artificiales: Características universales, lenguas filosóficas y lenguas secretas", Revista de Investigación Lingüística, 19, pp. 77-106.

Monnerot-Dumaine, M. (1960). Précis d'interlinguistique générale et spéciale. Paris: Maloine.

Peterson, D. J. (2014). Living Language Dothraki. Nueva Yok: IVY Books.

Peterson, D. J. (2015a). The art of language invention. From Horse-Lords to Dark Elves, the Words Behind World-Building. Nueva York: Penguin Books.

Peterson, D. J. (2015b). "The Languages of Ice and Fire". En Battis, J. y S. Johnston (eds.). Mastering the Game of Thrones: Essays on George R.R. Martin's A Song of Ice and Fire. Jefferson: McFarland.

Porset, C. (1979). "Langues nouvelles, langues philosophiques, langues auxiliaires au XXI siècle", Romantisme, 9 (25-26), pp. 209-215.

Violi, P. (1991). El infinito singular. Madrid: Cátedra.

Yaguello, M. (1984). Les fous du langage. Paris : Seuil. 